\title{
1946 \\ MEMBERS OF THE COUNCIL
}

\section{OFFICERS}

President.............................. H. HILDEBRANDT

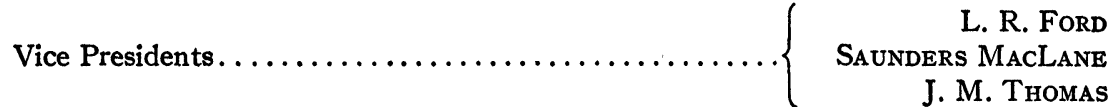

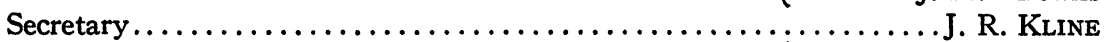

W. L. Ayres

R. H. BRUCK

Associate Secretaries.

T. R. HOLLCROFT

G. B. PRICE*

A. C. Schaeffer

Treasurer $\ldots \ldots \ldots \ldots \ldots \ldots \ldots \ldots \ldots \ldots \ldots \ldots \ldots \ldots \ldots \ldots \ldots \ldots \ldots \ldots$, P. GILL

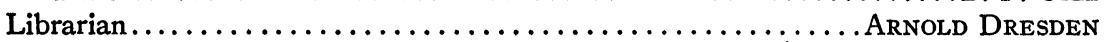

R. E. LANGER

Bulletin Editorial Committee..$\ldots \ldots \ldots \ldots \ldots \ldots \ldots$, SAUnders MacLANE

Deane MonTgomery

E. B. STOUFFER

A. A. Albert

Transactions Editorial Committee.

E. J. McShane

OSCAR ZARISKI

Mathematical Reviews Editorial Committee

O. E. Neugebauer M. H. STONE

Oswald Veblen

C. C. MACDuffee J. F. RitT

Colloquium Editorial Committee

Mathematical Surveys Editorial Committee.

G. T. WHYBURN

Nelson Dunford

A. W. TUCKER

J. L. WALSH

RICHARD BRAUER

L. M. Graves

Representatives on Board of Editors of the American Journal of Mathematics.

HAssler WhitNey

\section{Ex-Presidents}

G. C. Evans

MARston MORSE

M. H. Stone

\section{EX-SECRETARY}

R. G. D. RICHARDSON

\section{Members At Large}

To serve until

December, 1946

GARRETT BIRKHOFF

M. R. Hestenes

HaROLD Hotelling

Nathan Jacobson

R. D. JAMES

\section{To serve until}

December, 1947

H. F. BOHNENBLUST

S. S. CaIRNS

H. B. CURRY

M. H. INGRAHAM

I. S. SoKolNIKOFF
To serve until

December, 1948

R. P. BOAS

R. H. Cameron

R. V. Churchill

Churchill EisenharT

A. P. Morse

\section{BOARD OF TRUSTEES}

W. R. LONGLEY

MARSTON MORSE

\section{WARREN WEAver}

G. W. Mullins

R. G. D. RICHARDSON

* Succeeded W. L. Ayres, resigned September 1946. 